\title{
CUIDADOS PALIATIVOS: CONHECIMENTO DE ENFERMEIROS E APLICABILIDADE NO ÂMBITO HOSPITALAR
}

\section{PALLIATIVE CARE: KNOWLEDGE OF NURSES AND APPLICABILITY IN THE HOSPITAL FRAMEWORK}

Pollyana Bárbara Chimirri Desanoski', Bianca Machado Cruz Shibukawa ${ }^{1 *}$, Gabrieli Patricio Rissi', Juliana Dalcin Donini e Silva², leda Harumi Higarashi'

'Universidade Estadual de Maringá, Maringá, Paraná, Brasil.

${ }^{2}$ Centro Universitário de Maringá, Maringá, Paraná, Brasil.

*Autor correspondente: Universidade Estadual de Maringá, Maringá (PR), Brasil. Avenida Itororó, 1388. CEP: 87010-460. Telefone: (44) 99703-7149. E-mail: bih.cruvz@gmail.com.

\section{RESUMO}

A enfermagem é a profissão que lida com o cuidar, auxiliando a equipe multidisciplinar no processo de cuidado e reestabelecimento da saúde, todavia, nem sempre é possível alcançar a cura, principalmente quando se trata das neoplasias. Sendo assim, objetivou-se identificar os conhecimentos de enfermeiros em ambiente hospitalar sobre cuidados paliativos a pacientes oncológicos. Trata-se de um estudo descritivo, exploratório com abordagem qualitativa. A coleta dos dados se deu por meio de entrevistas gravadas e guiadas por questões norteadoras. Após, os depoimentos foram transcritos na íntegra garantindo a fidelidade dos dados, sendo assim meditadas, categorizadas e analisadas pelo método de análise de Bardin. Após inúmeras leituras, emergiram três categorias, sendo: a compreensão dos enfermeiros sobre cuidados paliativos; as dificuldades enfrentadas pelo profissional para implementação de cuidados paliativos no ambiente hospitalar e, a formação acadêmica do profissional sobre cuidados paliativos. Evidenciou-se que os enfermeiros se apoderam do conceito de cuidados paliativos de forma coerente e procuram colocá-lo em prática. Identificou-se que a maior necessidade percebida durante o cuidado é o conhecimento mais detalhado sobre a aplicabilidade da paliação. Apontou-se também a falta de preparo durante a graduação. Diante disso, considera-se que os enfermeiros conhecem a finalidade dos cuidados paliativos e desejam aplicá-lo, porém destacam a lacuna existente na formação, destacando a necessidade da abordagem desse tema de forma mais abrangente.

Palavras-chave: Cuidados paliativos; Enfermagem; Relações Profissional-Família; Enfermagem de Cuidados Paliativos na Terminalidade da Vida; Neoplasias.

\section{ABSTRACT}

Nursing is the profession that deals with caring, helping the multidisciplinary team in the process of health care and reestablishment; however, it is not always possible to achieve a cure, especially when it comes to neoplasms. Thus, the objective was to identify the knowledge of nurses in a hospital environment about palliative care for cancer patients. This is a descriptive, exploratory study with a qualitative approach. Data were collected through recorded interviews and guided by guiding questions. Afterwards, the statements were fully transcribed, guaranteeing the fidelity of the data, thus being meditated, categorized and analyzed by Bardin's analysis method. After numerous readings, three categories emerged, namely: nurses' understanding of palliative care; 
the difficulties faced by the professional to implement palliative care in the hospital environment and the academic education of the professional about palliative care. It was evidenced that nurses take the concept of palliative care coherently and try to put it into practice. It was identified that the greatest perceived need during care is the more detailed knowledge about the applicability of palliation. It was also pointed out the lack of preparation during graduation. Given this, it is considered that nurses know the purpose of palliative care and want to apply it, but highlight the gap in training, highlighting the need to address this issue more broadly.

Keywords: Palliative Care; Nursing; Professional-Family Relations; Hospice and Palliative Care Nursing; Neoplasms.

\section{INTRODUÇÃO}

As neoplasias representam a segunda causa de mortalidade em âmbito mundial, ultrapassando apenas as doenças cardiovasculares, embora existam projeções de estas ocuparem o primeiro lugar em países desenvolvidos nos próximos anos ${ }^{1}$.

De acordo com o Associação Norte-Americana de Registros Centrais de Câncer surgiram 1.688.780 novos casos de câncer, em 2017, com projeções de 600.920 mortes pela doença nos Estados Unidos $^{2}$. Estima-se que até 2025 haja 25 milhões de novos casos de câncer anualmente ${ }^{3-4}$.

Segundo a última estimativa do Instituto Nacional de Câncer José Alencar Gomes da Silva (INCA), o Brasil apresentou cerca de 576 mil casos novos de câncer em 2015, sendo que, com exceção ao câncer de pele do tipo não melanoma, as principais manifestações da doença foram referentes a tumores de próstata, mama feminina, cólon e reto, pulmão, estômago e colo do útero ${ }^{5}$.

Considerando o elevado número de pacientes acometidos pelas neoplasias, assim como suas elevadas taxas de mortalidade, faz-se necessário considerar os cuidados paliativos como forma de melhorar a qualidade de vida dos indivíduos e famílias frente ao enfrentamento desta condição ${ }^{6}$.

O cuidado paliativo visa diminuir o sofrimento humano diante de uma situação incurável, por meio de sentimentos e comunicação empáticos, objetivando fornecer apoio e estimular a autonomia do indivíduo para lidar com situações ameaçadoras da vida ${ }^{6-7}$.

Diante disso, os cuidados paliativos são indispensáveis, à medida que visam melhorar a qualidade de vida do paciente e de seus familiares, quando este, não possui mais resposta ao processo curativo e o prognóstico não é mais positivo.

A enfermagem auxilia a equipe multidisciplinar no processo de cuidado e reestabelecimento da saúde, todavia, nem sempre é possível alcançar a cura, principalmente quando se trata das neoplasias, sendo assim, torna-se importante questionar o preparo desses profissionais na assistência ao paciente oncológico.

Logo, há necessidade de empenho pela equipe de saúde, por meio do trabalho interprofissional, atendendo às necessidades de cuidado do cliente e da família diante das incertezas e dificuldades vivenciadas com a instabilidade do quadro clínico do cliente e a proximidade da morte ${ }^{8}$. 
Portanto, o objetivo do trabalho foi identificar os conhecimentos de enfermeiros em ambiente hospitalar sobre cuidados paliativos a pacientes oncológicos e as dificuldades desses profissionais em executar os cuidados paliativos.

\section{METODOLOGIA}

Trata-se de uma pesquisa descritiva, exploratória com abordagem qualitativa. Foram sujeitos do estudo nove enfermeiros que atuavam nos setores de clínica médica e Unidade de Terapia Intensiva (UTI) em um hospital público do Noroeste do Paraná.

Os critérios de inclusão para participação do estudo foram profissionais enfermeiros que atuassem minimamente há seis meses nestes setores. Foram excluídos do estudo os profissionais que estavam de férias, licença ou atestado médico e aqueles que se recusaram a participar.

A coleta de dados ocorreu em 2015. Para realização da mesma, foi utilizado um questionário semiestruturado contendo dados de identificação e questões norteadoras, guiadas por entrevistas, as quais foram gravadas e transcritas na íntegra, garantindo a fidelidade dos depoimentos.

Primeiramente foi realizado contato telefônico com os enfermeiros, onde foram expostos os objetivos da pesquisa. Perante a aceitação da participação no estudo, foram agendados data, horário e local de preferência dos sujeitos para realização das entrevistas.

Em dia e hora marcados, foi apresentado o Termo de Consentimento Livre e Esclarecido (TCLE), que após leitura e compreensão, que foi assinado pelo depoente e pesquisador em duas vias, sendo uma delas entregue ao participante e outra arquivada.

Para análise dos dados, os depoimentos foram meditados, categorizados e analisados pelo método de análise de conteúdo de Bardin'.

O estudo foi aprovado pelo Centro de Capacitação de Profissionais da Saúde de Maringá (CECAPS) e pelo Comitê de Ética e Pesquisa (CEP) da Unicesumar. Ressalta-se que foram respeitados todos os princípios éticos e legais estabelecidos pelas Resoluções 466/2012 $2^{11}$, 510/2016 ${ }^{12}$ e 580/2018 ${ }^{13}$ do Conselho Nacional de Saúde.

\section{RESULTADOS E DISCUSSÕES}

Participaram da pesquisa nove enfermeiros que atuam no setor de clínica médica e Unidade de Terapia Intensiva (UTI), em um hospital público da região noroeste do Paraná. A idade média dos participantes variou entre 27 e 48 anos de idade. Em relação ao tempo de formação dos entrevistados, verificou-se que o mínimo correspondeu a 5 anos e o tempo máximo a 25 anos, sendo que todos alegaram que trabalharam desde a graduação, o que foi verificado por um tempo de serviço médio de 14 anos.

Após realização de inúmeras leituras acerca dos depoimentos obtidos, emergiram três categorias temáticas, sendo elas: a compreensão dos enfermeiros sobre cuidados paliativos; as dificuldades enfrentadas pelo profissional para implementação de cuidados paliativos no ambiente hospitalar e, a formação acadêmica do profissional sobre cuidados paliativos.

\section{A compreensão dos enfermeiros sobre cuidados paliativos}

Pensando na melhoria da qualidade de vida de pessoas com doenças progressivas e fora de possibilidades terapêuticas, os profissionais buscam a implementação de um cuidado diferenciado 
no cotidiano dos serviços de saúde. Neste cenário, os cuidados paliativos passaram a fazer parte do cotidiano dos serviços de saúde existentes no Brasil ${ }^{13}$.

O cuidado paliativo, dentro da medicina paliativa, não tem por intuito nem acelerar tão pouco retardar o processo natural da morte; mas o reconhece como um processo natural e progressivo para todos ${ }^{14}$. Nesta perspectiva, procura-se dispor todo apoio e ajuda ao paciente, para que ele consiga viver mais ativamente possível até a hora de sua morte; nesse mesmo pensar, os familiares são encorajados a vivenciarem esse momento com mais naturalidade, com diminuição do sofrimento diante da doença e processo de morte do ente querido ${ }^{15}$.

Observando o cenário de saúde atual em relação as doenças crônicas e em especial as neoplasias, foi possível verificar nos depoimentos abaixo, a compreensão dos enfermeiros sobre cuidados paliativos.

Aqueles cuidados que a gente presta ao paciente para alivio da dor, para conforto, para os pacientes que não tem prognostico, que na verdade estão aguardando uma morte. Cuidados paliativos, vão fazer com que ele tenha uma morte mais digna, com menos sofrimento. ( E1)

São aqueles pacientes que já estão em fase terminal e você vai proporcionar um conforto para ele, então esses cuidados paliativos é você proporcionar um conforto, você retirar a dor dele. (E2)

Cuidados paliativos são aqueles cuidados que você dispensa aos pacientes né, quando pela medicina todos os recursos que temos disponíveis já não serão benéficos ao paciente. (E3)

Os relatos dos depoentes vão de encontro com a literatura, onde cuidados paliativos podem ser também denominados como cuidados de fim de vida, que nasceram, a princípio, para atender aos pacientes portadores de neoplasia em estágio avançado, todavia, foram estendidos a todo paciente portador de alguma doença que cause dor intensa, sintomas físicos, sofrimento emocional e espiritual profundos, tornando a vida quase insuportável ${ }^{16}$.

Os cuidados paliativos são uma filosofia desenvolvida por Cicely Sauders em 1960, no qual descreveu também o conceito de dor total como sendo a dor física, psicológica, social e espiritual. Essa filosofia busca afirmar a vida e encarar a morte como um processo natural, não apressando e nem adiantando a morte, aliviar a dor e dar apoio tanto para a pessoa em sofrimento quanto à família ${ }^{17}$.

Nesse pensar, o conhecimento dos profissionais de saúde e, de forma especial, dos enfermeiros, torna-se fundamental para a efetivação do cuidado idealizado e praticado por Cicely Sauders.

\section{As dificuldades enfrentadas pelo profissional para implementação de cuidados paliativos no ambiente hospitalar}

Acredita-se que um dos maiores problemas enfrentados pelas equipes de saúde ao tratar dos pacientes em cuidados paliativos é a falta de conhecimento sobre a aplicabilidade da paliação, que, segundo a Organização Mundial de Saúde, consiste na melhoria da qualidade de vida do paciente e seus familiares, diante de uma condição que ameace a vida, por meio da prevenção e alívio do sofrimento, da identificação precoce, avaliação e tratamento de dor e demais sintomas físicos, sociais, psicológicos e espirituais ${ }^{18}$.

A magnitude dos termos "cuidado total" e "ativo" representam a exata dimensão da visão sobre cuidados paliativos, cuidado no sentido multidimensional, considerando as necessidades destes pacientes em todos os seus aspectos e, ativo, no sentido do afastamento da passividade e investimentos pelo aprimoramento e qualificação da assistência ${ }^{19}$. 
Procura-se enfatizar a importância dos sintomas psicológicos, espirituais e sociais, aumentando as responsabilidades da assistência, que deve atuar além do controle de sintomas físicos, priorizando o alívio do sofrimento humano e proporcionando a melhoria da qualidade de vida diante de sua experiência desvelada no enfrentamento da doença ${ }^{18-19}$.

Nessa mesma perspectiva, o relato a seguir mostra a preocupação do profissional com a dor do paciente em cuidados paliativos.

O alivio da dor acho que é a principal necessidade, porque dependendo de onde for o câncer o paciente sente muita dor mesmo né? E a questão do psicológico também de alguns pacientes, que permanecem conscientes e orientados, mesmo durante os cuidados paliativos e precisam desse suporte. (E4)

A dor acomete 60 a 80 \% dos pacientes com câncer sendo 25 a 30\% na ocasião do diagnóstico e 70 a $90 \%$ dos pacientes com doença avançada classificam a dor como moderada a grave. Diante desses fatos a Organização Mundial de Saúde (OMS) declarou a dor associada ao câncer uma Emergência Médica Mundial publicando em 1986 um guia de tratamento que pode proporcionar alívio da dor em $90 \%$ dos pacientes ${ }^{20}$.

O sucesso da terapia da dor no paciente com câncer baseia-se principalmente no diagnóstico do mecanismo da dor (inflamatório, neuropático, isquêmico, compressivo) e consequentemente do diagnóstico da síndrome dolorosa preponderante ${ }^{20}$.

Respeitar a autonomia do paciente favorece confiança na relação terapêutica entre os profissionais e os pacientes, fortalecendo os laços de cooperação ao tratamento e satisfação com relação ao acompanhamento. Em termos legais, respalda os direitos individuais sobre o próprio corpo, além de ofertar ao paciente o senso de controle sobre a própria vida e de valor pessoal ${ }^{21}$.

Comunicar-se adequadamente com o paciente e seus familiares torna-se uma medida eficaz para o cuidado prestado ao paciente, reduzindo as angústias, o estresse e a ansiedade ao compartilhar o sofrimento com a equipe ${ }^{22}$.

Portanto, é fundamental que os profissionais voltem seu olhar atento ao outro, prestando atenção em seus gestos, fala e atitudes e assim, estabelecer uma relação de confiança com paciente e família.

Eu vejo que a necessidade maior assim, é a família, porque eu acho que e nesse momento, em que eles mais precisam da família, e as vezes, a família não tem estrutura psicológica, não entendem o que está acontecendo acabam deixando desejar em relação a isso. (E5)

Então eu acho que a nossa grande falha e a falta de envolvimento da família. Porque na verdade quando o paciente está doente, e a gente vai vendo que ele não vai melhorando e que o prognóstico dele não está bom, eu acho que a gente demorar muito pra envolver a família nesse processo, então quando se fala que é cuidados paliativos, pensamos que a família já está preparada, e já quer mandar por exemplo o paciente da UTI, ou para enfermaria, explicar pra família, acredito que desde o momento da internação, já deveríamos começar a explicar pra família tudo, então eu acho que nossa falha está nesse ponto, assim de demorar para envolver a família[...].' (E3)

A participação e envolvimento da família no cuidado ao paciente em cuidados paliativos é fundamental para a melhora do estado geral, pois é o familiar que convive e conhece o doente em suas vontades e necessidades. Logo, a fragilidade do momento, muitas vezes, impede a família de cuidar adequadamente, situação muitas vezes incompreendida pelos profissionais de enfermagem, que direcionam críticas a família sem ao menos conhecê-la ${ }^{23}$. 
Por outro lado, em inúmeras vezes o profissional de enfermagem também se sente impotente diante das situações experiências com pacientes em cuidados paliativos e suas famílias, impotência decorrente da falta de conhecimento ou pela falta de apoio da equipe interdisciplinar ${ }^{24}$. Os profissionais entrevistados têm ciência de que os cuidados precisam ser melhorados, como no relato abaixo.

A gente poderia fazer mais coisa, mais intervenções como por exemplo alivio mesmo, bolsa de agua quente mudança de decúbito, ciosas não farmacológicas, nós poderíamos fazer mais, não e feito tudo que a gente pode eu acho, a gente não usa toda nossa capacidade, todos os nossos conhecimentos. (E1)

Este sentimento que angustia o profissional e o faz pensar que poderia fazer mais pelo paciente na terminalidade da vida, foi descrito na literatura, onde os profissionais sentem-se despreparados e impotentes para realização dos cuidados nessa fase $\mathrm{f}^{23-24}$.

\section{A formação acadêmica do enfermeiro sobre cuidados paliativos.}

Atuar no campo dos cuidados paliativos exige conhecimento técnico-científico, além de constante enfrentamento da morte e de suas implicações do processo de morrer, portanto os profissionais desta área precisam desenvolver empatia ao sofrimento do próximo, comumente pouco trabalhadas nos cursos da área da saúde prejudicando a formação de novos profissionais ${ }^{25}$.

A morte sempre foi e continuará a ser um grande desafio para os profissionais da área da saúde. A diferença é que para profissionais desta área, como médicos, enfermeiros e psicólogos, a morte passa a fazer parte do cotidiano e pode se tornar sua companheira diária de trabalho ${ }^{23-24}$.

Com base nas necessidades em que os profissionais enfrentam no dia a dia, relataram a dificuldades da implementação dos cuidados paliativos, assim como o despreparo profissional pela falta de orientação durante a academia.

Uma disciplina não tive, uma vez tive uma palestra a respeito, bem superficial, foi questão assim de uma hora uma hora e meia falando sobre o assunto, mas foi só, só isso. (E1)

Não, não tive. Não sei se na época que me formei também não tinha muito diagnóstico de câncer como tem hoje. (E4)

De semelhante forma, um estudo ${ }^{26}$ demostrou que apenas $22 \%$ dos enfermeiros entrevistados disseram que tiveram alguma informação sobre cuidados paliativos durante a graduação, e 33\% responderam que aprenderam com a prática após a formação.

Logo, as respostas dos participantes foram convergentes, onde percebeu-se que todos os profissionais encontram as mesmas dificuldades em trabalhar com cuidados paliativos.

Na verdade a gente acaba aprendendo com a vida, aprendendo no dia a dia ali ne? Porque base mesmo a gente não tem na formação. (E2);

Acho falho, tem que ter na graduação pelo menos uma disciplina, né. Que ensinasse o aluno, por exemplo, o que eu sei de cuidados paliativos foi depois de formada, mas durante a graduação não, foi bem precário. (E1)

A educação para a morte é necessária, visto que, os profissionais em sua prática diária distanciam-se do assunto realizando atividades rotineiras dando ênfase às técnicas realizadas com os pacientes, evidenciando o despreparo frente a morte devido a questões culturais, espirituais e falta de ensino durante a academia, que ainda enfatiza a formação tecnicista, contrariando os aspectos emocionais, sociais e espirituais ${ }^{27}$. 
A formação dos profissionais da saúde em cuidados paliativos deve abranger e estimular o desenvolvimento de habilidades entre uma equipe multidisciplinar para o suporte técnico de enfrentamento de morte e luto a pacientes, familiares e profissionais da saúde 26-27. $^{2}$.

\section{CONCLUSÕES}

Evidenciou-se que os enfermeiros reconhecem suas falhas e falta de conhecimento sobre o assunto, limitando o processo de cuidado de acordo com os princípios estabelecidos por Cicely Sauders que são, até hoje, a essência dos cuidados paliativos.

Os depoimentos sobre a formação profissional para enfrentamento do processo de morte/ morrer convergiram para a falta de preparo oferecido nos cursos de graduação, dificultando o agir do enfermeiro no dia-a-dia.

É importante destacar que o enfermeiro é um dos principais atores na luta pelos cuidados que visam a melhoria da qualidade de vida de pacientes, tornando-se essencial que as instituições de ensino superior invistam em disciplinas voltadas ao cuidado de pessoas mediante a impossibilidade da cura e proximidade com a morte.

\section{REFERÊNCIAS}

1. Oliveira MM, Malta DC, Guauche H, Moura L, Silva GA. Estimated number of people diagnosed with cancer in Brazil: data from the National Health Survey, 2013. Rev Bras Epidemiol [Internet]. 2015 [acesso em 2019 abr 3]; 18 (2): 146-157. Disponível em: http://www.scielo.br/scielo. php?script=sci_arttext\&pid=S1415-790X2015000600146.

2. Siegel RL, Miller KD, Jemal A. Cancer Statistics, 2017. CA Cancer J Clin [Internet]. 2017 [acesso em 2019 abr 3]; 67(1):7-30. Disponível em: http://dx.doi. org/10.3322/caac.21387.

3. Ferlay J, Soerjomataram I, Dikshit R, Eser S, Mathers C, Rebelo M, et al. Cancer incidence and mortality worldwide: sources, methods and major patterns in GLOBOCAN 2012. Int J Cancer [Internet]. 2015 [acesso em 2019 abr 3]; 136(5):E359-86. Disponível em: http://dx.doi.org/10.1002/ ijc.29210.

4. Gayoso MV, Avila MAG, Silva TA, Alencar RA. Comfort level of caregivers of cancer patients receiving palliative care. Rev Latino-Am Enfermagem [Internet]. 2018 [acesso em 2019 abr 3]; 26:e3029. Disponível em: http://dx.doi.org/ 10.1590/1518-8345.2521.3029.

5. Instituto Nacional de Câncer. José Alencar Gomes da Silva. Coordenação de Prevenção e Vigilância. Estimativa 2014: Incidência de Câncer no Brasil / Instituto Nacional de Câncer José Alencar Gomes da Silva, Coordenação de Prevenção e Vigilância. Rio de Janeiro: INCA, 2014, $124 p$.

6. Arrieira ICO, Thofehrn MB, Porto AR, Moura PMM, Martins CL, Jacondino MB. Spirituality in palliative care: experiences of an interdisciplinary team. Rev Esc Enferm USP [Internet]. 2018 [acesso em 2019 abr 3]; 52:e03312. Disponível em: http://www.scielo.br/scielo.php?script=sci_ arttext\&pid=S0080-623420180001 $00401 \&$ Ing=en\&nrm=iso\&tlng=em.

7. Gulini JEHMB, Nascimento ERP, Moritz RD, Rosa LM, Silveira NR, Vargas MAO. Intensive Care Unit team perception of palliative care: the discourse of the collective subject. Rev Esc Enferm USP [Internet]. 2017 [acesso em 2019 abr 3]; 51 :e03221. Disponível em: https://www.ncbi.nlm.nih. gov/pubmed/28562740. 
8. Silva MM, Moreira MC, Leite JL, Erdmann AL. Evidence of care comprehensiveness in the practice of nursing staff in oncology palliative care. Rev Eletr Enf [Internet]. 2014 [acesso em: 24 set 2018]; 16(4):795-803. Disponível em: http://dx.doi.org/10.5216/ree.v16i4.25700.

9. Bardin, L. Análise de conteúdo. São Paulo: Edições 70, 2011 ,229p.

10. BRASIL. Conselho Nacional de Saúde. Resolução n 466, de 16 de dezembro de 2012. Diário Oficial da União, Brasília-DF, jun. 2013; Seção 1. Disponível em: http://conselho.saude.gov.br / resolucoes/2012/reso466.pdf.

11. BRASIL. Conselho Nacional de Saúde. Resolução n 510, de 07 de abril de 2016. Diário Oficial da União, Brasília-DF, mai. 2016; Seção 1. Disponível em: http://conselho.saude.gov.br/resolucoes/ 2016/reso510.pdf.

12. BRASIL. Conselho Nacional de Saúde. Resolução n 580, de 22 de março de 2018. Diário Oficial da União, Brasília-DF, mar. 2018; Seção 1. Disponível em: http://www.cofen.gov.br/resolucaocofen-no-580-2018_64035.html

13. Carvalho RT, Parsons HA. Manual De Cuidados Paliativos. Academia Nacional De Cuidados Paliativos. 2 ed., 2012.

14. Euzébio MR, Cruz ICF. Nursing evidence-based inteprofissional practice guidelines on palliative care and ICU-related death anxiety - systematic literature review. Journal Specialized Nursing Care [Internet]. 2019 [acesso em: 03 abr 2019]; 11(1). Disponível em: http://www.jsncare.uff.br/ index.php/jsncare/article /view/3181/8 07.

15. Freitas TLL, Banazeski AC, Eisele A, Souza EN, Bitencourt JVOV, Souza SS. The look of nursing on death and dying process of critically ill patients: an integrative review. Enfermería Global. 2016; 41:335-347.

16. Carvalho GAFL, Menezes RMP, Enders BC, Teixeira GA, Dantas DNA, Oliveira DRC. Significados atribuidos por los profesionales de la salud para los cuidados paliativos en el contexto de la atención primaria. Texto \& Contexto [Internet]. 2018 [acesso em: 03 abr 2019]; 27(2): e5740016. Disponível em: http://ciberindex.com/c/tc/27222p.

17. Castro EK, Barreto SM. Oncology medical criteria for psychologycal referral in palliative care. Psicologia: ciência e profissão [Internet]. 2015 [acesso em: 03 abr 2019]; 35(1),69-82. Disponível em: http://www.scielo.br/pdf/pcp/v35n1/1414-9893-pcp-35-01-00069.pdf

18. World Palliative Care Alliance; World Health Organization. Global Atlas of Palliative Care at the End of Life. Geneva: World Health Organization. 2014.

19. Semtchuck ALD, Genovesi FF, Santos JL. Los cuidados paliativos en oncología pediátrica: revisión integradora. Rev Uruguaya de Enfermería [Internet]. 2017 [acesso em: 03 abr 2019]; 12(1). Disponível em: http://rue.fenf.edu.uy/ index.php/rue/article/view/216

20. Rangel O, Telles C. Tratamento da Dor Oncológica em Cuidados Paliativos. Rev Hosp Univers Pedro Ernesto, UERJ [Internet]. 2012 [acesso em: 24 out 2018]; 11:32-37. Disponível em: http:// revista.hupe.verj.br/detalhe_artigo.asp?id=324.

21. Abreu CBB, Fortes PAC. Questões éticas referentes às preferências do paciente em cuidados paliativos. Rev Bioét [Internet]. 2014 [acesso em: 03 abr 2019]; 22 (2): 299-308. Disponível em: http://dx.doi.org/10.1590/1983-80422014222011. 
22. Ambrósio DCM, Santos MA. Apoio social à mulher mastectomizada: um estudo de revisão. Ciênc saúde coletiva [Internet]. 2015 [ acesso em: 03 abr. 2019]; 20 (3):851-864. Disponível em: http://dx.doi.org/10.1590/1413-81232015203. 13482014.

23. Silva RS, Trindade GSS, Paixão GPN, Silva MJP. Conferência familiar em cuidados paliativos: análise de conceito. Rev Bras Enferm [Internet]. 2018 [citado em: 03 abr 2019]; 71 (1): 206-213. Disponível em: http://dx.doi.org/10.1590/ 0034-7167-2016-0055.

24. Bastos RA, Lamb FA, Quintana AM, Beck CLC, Carnevale F. Vivências dos enfermeiros frente ao processo de morrer: uma metassíntese qualitativa. Rev Portuguesa de Enfermagem de Saúde Mental [Internet]. 2017 [citado em: 03 abr 2019];17: 58-64. Disponível em: http://dx.doi. org/10.19131/rpesm.0184.

25. Santos ALN, Lira SS, Costa RSL. Palliative cares provided by the nurse to the oncological patient. Dê Ciência em Foco. 2018; 2(1): 63-77.

26. Fortunado AMO, Souza SROS, Ramos SJ, Ferreira MC. O Enfermeiro frente ao paciente fora da possibilidade terapêutica: dignidade e qualidade no processo de morrer. Enfermería Global. $2011 ; 22: 1-7$.

27. Santos JL, Corral-Mulato S, Bueno SMV. Morte e luto: a importância da educação para o profissional de saúde. Arq Cienc Saúde UNIPAR [Internet]. 2014 [citado em: 03 abr 2019]; 18(3):199-203. Disponível em: http://www.revistas.unipar.br/index. php/saude/article/ viewFile/5196/3008 